\title{
Omitting Nasogastric Tube Placement after Gastrectomy Does Not Enhance Postoperative Recovery: A Propensity Score Matched Analysis
}

\section{Zhouqiao Wu}

Beijing Cancer Hospital

\section{Qi Wang}

Beijing Cancer Hospital

Jinyao Shi

Beijing Cancer Hospital

\section{Shiyang Hou}

Beijing Cancer Hospital

\section{Fei Shan}

Beijing Cancer Hospital

\section{Shuangxi Li}

Beijing Cancer Hospital

\section{Yan Zhang}

Beijing Cancer Hospital

Ziyu Li ( $\square$ ziyu_li@hsc.pku.edu.cn )

Peking University Cancer Hospital https://orcid.org/0000-0001-9222-181X

Jiafu Ji

Beijing Cancer Hospital

\section{Research article}

Keywords: Nasogastric tube, Gastric tumor, Postoperative complications, Length of postoperative hospital stay

Posted Date: March 11th, 2020

DOl: https://doi.org/10.21203/rs.3.rs-16585/v1

License: (c) (1) This work is licensed under a Creative Commons Attribution 4.0 International License.

Read Full License 


\section{Abstract}

Background: Enhanced recovery after surgery (ERAS) program has become the main trend in gastrointestinal surgery. The aim of this study is to investigate factors influencing the decision-making of nasogastric tube (NGT) placement and its safety and efficacy in clinical practice.

Methods: We analyzed our prospectively maintained database including 287 patients underwent elective gastrectomy in our department from January 1 to December 31, 2017. All cases were divided into two groups, namely, the non-nasogastric tube group and the nasogastric tube group. Logistic regression was used to analyze the factors that affect the decision of nasogastric tube placement, and propensity score matching (PSM) was later applied to balance those factors for the analysis of the safety outcomes between the groups.

Results: Multivariate analysis showed that resection range ( $p=0.004$, proximal gastrectomy: $O R=4.555$, $95 \% \mathrm{Cl}=1.392-14.905, \mathrm{p}=0.016$; total gastrectomy: $\mathrm{OR}=1.990,95 \% \mathrm{Cl}=1.205-3.287, \mathrm{p}=0.009$ ) was the only independent risk factors of nasogastric tube placement. NGT was omitted in the majority $(58.8 \%)$ of distal gastrectomy, but only in $42.5 \%$ and $25 \%$ in total and proximal gastrectomy. After PSM, we found no significant differences between patients with or without NGT in postoperative hospital stay, time to first flatus and defecation, time to fluid and semi-fluid diet, rate of reinsertion, or hospitalization expenditure ( $p>0.05$ respectively). The incidence of postoperative complications in the two groups were $21.7 \%$ and $23.5 \%$ respectively $(p=0.753)$, and the incidence of major complications were $7.0 \%$ and $9.6 \%(p=0.472)$.

Conclusions: The decision-making of NGT placement is mainly influenced by the resection range. Omitting NGT is a safe approach in all types of gastrectomy but was not able to enhance the recovery in our practice.

\section{Background}

Ever since Kehlet first proposed the concept of Enhanced Recovery After Surgery (ERAS) in 1997, it has been carried out successfully in varied surgical fields. Nowadays, rapid recovery after operations has gradually become clinical routine in gastrointestinal surgery. With the deep understanding of the rapid recovery after gastrectomy, many studies question the necessity of nasogastric tube (NGT) after gastrectomy [1-3]. The international guidelines [4] and domestic expert consensus [5] recommend that there is no need to insert nasogastric tube routinely in patients with gastrectomy, and several randomized controlled trials (RCT) $[6-10]$ and meta-analyses $[1,11]$ have provided strong evidences against the routine use of nasogastric tube following gastrectomy.

However, nasogastric tube is routinely used in the majority, if not all, gastric cancer patients after gastrectomy in China. With the accumulating evidence against the routine use of nasogastric tube, it is yet unclear that what factors exactly influence the decision making of nasogastric tube placement in China. Moreover, whether omitting nasogastric tube is safe in the setting of Chinese clinical practice also requires more evidence for verification. Since 2017, our department has gradually educated the ERAS 
program in gastric cancer patients. Surgeons were also encouraged to avoid the tube placement when consider safe. In this study, we retrospectively analyzed our prospectively maintained database to explore the influence factors of the decision making of nasogastric tube placement, to evaluate whether omitting nasogastric tube accelerate the postoperative recovery of patients after gastrectomy, and last but not least, to assess whether it is safe after gastrectomy without nasogastric tube.

\section{Methods}

\section{Patient and Clinical data registration}

In this study, we included patients underwent elective gastrectomy for gastric tumor in the ward I of gastrointestinal cancer center, Peking University Cancer Hospital \& Institute from January 1, 2017 to December 31, 2017. During that time, placing nasogastric tube were no longer restricted as clinical routine and the surgeons might choose not to place the tube when they thought it was safe to do so. We collected the patients' demographic data, clinical pathological characteristics, and perioperative information from a prospectively maintained database [12]. Moreover, since 2017, we prospectively registered the postoperative complication data in accordance to the APPEAL study protocol [13-14] and the severity of complications were scored using the Clavien-Dindo grading system [15] continuously by one set clinical researcher. The postoperative complication data were retrieved from this database to ensure the objectiveness and quality of the data.

\section{Perioperative managements}

The perioperative managements of patients were consistent in accordance with our ERAS protocol. In short, one day prior to the operation, laxative was applied to the patients with no additional mechanical bowel preparation. Patients were fasted from solid food for $12 \mathrm{~h}$, while they were suggested to drink $250 \mathrm{ml} \mathrm{10 \%} \mathrm{glucose} \mathrm{in} \mathrm{the} \mathrm{morning} \mathrm{of} \mathrm{the} \mathrm{operation.} \mathrm{Prophylactic} \mathrm{antibiotics} \mathrm{were} \mathrm{administrated} \mathrm{for} \mathrm{one}$ dose before the operation and 1-2 doses afterwards during perioperative period (in total within $24 \mathrm{~h}$ ). After surgery, patient-controlled analgesia (PCA) was given to patients for the first three postoperative days, and additional non-steroidal anti-inflammatory drugs or opioids were administrated when acute pain occurred accordingly. In general, patients were recommended to drink water (in small amount) on the first day after surgery, and the total amount of food intake gradually increased daily after surgery based on the patient's feeling and the doctor's judgment. Patients were allowed to take soft food if they have good tolerance of liquid or their bowel function was recovered. We also encouraged them to ambulate at an early stage, usually at the first day after surgery. The patients were discharged when they met the following criteria: satisfied mobility, tolerance of soft food intake for at least one day, not suspicious of complications [5].

\section{Study endpoints}

The major purpose of our study is to determine whether omitting the nasogastric tube accelerates the postoperative recovery in our clinical setting. Therefore we choose the primary endpoint as the length of 
postoperative hospital stay, which was defined as the day from the first day after surgery to the day of discharge (including the day of discharge).

To achieve this, we first investigated what are the major factor that influence the decision-making of NGT placement by logistic regression, and then a propensity score matching (PSM) was performed to control the influence of the identified independent risk factor(s) between the groups. In the regression analysis, we included the patient characteristics and intraoperative data into the univariate and multivariate analyses, which were performed by non-conditional logistic regression analysis, and the factors with predictive value in univariate analysis were included in multivariate analysis. The nearest neighbor method was used in the PSM, and caliper width was set to 0.1. The clinical features between the two groups were compared after PSM.

The other recovery outcomes include the time to first flatus and defecation, the time to fluid and semifluid diet, and the rate of tube reinsertion. The hospitalization expenses, as one major advantage of the ERAS program, was also measured. In addition to the recovery outcomes, we also measured the safety outcomes (i.e. postoperative complications) between the two corrected groups. The diagnosis and classification of complications was based on Clavien-Dindo classification, and we define those grade III or higher complication as severe complication [15]. The complications included in this study and the diagnostic criteria were referred to the APPEAL study [13-14], and were first recorded by the doctors and double checked by a set researcher. The study design is shown in Fig. 1.

\section{Statistical analysis}

In statistical analysis, the continuous variables such as age, blood loss and postoperative hospital stay were described as the mean \pm standard deviation if kolmogorov-smirnov test was consistent with a normal distribution. Otherwise, the median were used. For the classified variables such as gender, TNM stage, and tumor location, we described the number of cases and percentage. Differences in variables among groups were tested using student's t test, chi-square test, or Fisher's exact test. However, nonparametric tests (the Krukal-Wallis or Mann-Whitney test) were used for variables with skewed distributions. Bilateral $p<0.05$ was considered statistically significant. We used SPSS version 24.0 (IBM SPSS Software) for all statistical analyses.

\section{Results}

\section{Patient characteristics}

After the exclusion of patients who had previous history of gastric surgery, emergency surgery (acute perforation, obstruction, bleeding, etc.), we included 287 patients underwent gastrectomy for gastric tumor in our ward of Peking University Cancer Hospital \& Institute from January 1, 2017 to December 31, 2017. These cases were divided into two groups, namely, the no-NGT group $(n=147)$ and the NGT group 
$(n=140)$. The patients' demographic data (Table 1.1), clinical pathological characteristics (Table 1.2), and perioperative information (Table 1.3) are summarized in Table 1. 
Table 1.1

Demographic data of the patients

\begin{tabular}{|c|c|c|c|c|}
\hline & no-NGT $(N=147)$ & NGT $(N=140)$ & Total $(\mathrm{N}=\mathbf{2 8 7})$ & $P$ value \\
\hline Gender & & & & 0.445 \\
\hline Male & $111(75.5)$ & 111(79.3) & 222(77.4) & \\
\hline Female & $36(24.5)$ & $29(20.7)$ & $65(22.6)$ & \\
\hline Age & & & & 0.836 \\
\hline$<65$ & $97(66)$ & $94(67.1)$ & 191(66.6) & \\
\hline$\geq 65$ & $50(34)$ & $46(32.9)$ & $96(33.4)$ & \\
\hline City & & & & 0.207 \\
\hline Beijing & $34(23.1)$ & $24(17.1)$ & $58(20.2)$ & \\
\hline Others & 113(76.9) & 116(82.9) & $229(79.8)$ & \\
\hline BMI (kg/囚) & & & & 0.711 \\
\hline$<18.5$ & $5(3.4)$ & $5(3.6)$ & $10(3.5)$ & \\
\hline$[18.5,25.0)$ & 88(59.9) & $92(65.7)$ & $180(62.7)$ & \\
\hline$[25,30)$ & $47(32.0)$ & $36(25.7)$ & 83(28.9) & \\
\hline$\geq 30$ & $7(4.8)$ & $7(5.0)$ & $14(4.9)$ & \\
\hline Preoperative comorbidity & & & & 0.873 \\
\hline No & $90(61.2)$ & $87(62.1)$ & 177(61.7) & \\
\hline Yes & $57(38.8)$ & $53(37.9)$ & $110(38.3)$ & \\
\hline Abdominal surgery history & & & & 0.333 \\
\hline No & 114(77.6) & 115(82.1) & $229(79.8)$ & \\
\hline Yes & $33(22.4)$ & 25(17.9) & $58(20.2)$ & \\
\hline Smoke & & & & 0.248 \\
\hline No & 73(49.7) & $60(42.9)$ & $133(46.3)$ & \\
\hline Yes & $74 f(50.3)$ & $80(57.1)$ & 154(53.7) & \\
\hline Alcohol & & & & 0.239 \\
\hline No & $95(64.6)$ & $81(57.9)$ & 176(61.3) & \\
\hline
\end{tabular}

NGT nasogastric tube, BMI body mass index, NRS2002 nutritional risk screening 2002 


\begin{tabular}{|lclll|}
\hline & no-NGT (N=147) & NGT (N=140) & Total (N=287) & P value \\
\hline Yes & $52(35.4)$ & $59(42.1)$ & $111(38.7)$ & \\
\hline NRS2002 & & & 0.454 \\
\hline$<3$ score & $127(86.4)$ & $125(89.3)$ & $252(87.8)$ & \\
\hline$\geq 3$ score & $20(13.6)$ & $15(10.7)$ & $35(12.2)$ & \\
\hline NGT nasogastric tube, BMI body mass index, NRS2002 nutritional risk screening 2002 & \\
\hline
\end{tabular}


Table 1.2

Clinical pathological characteristics of the patients

\begin{tabular}{|c|c|c|c|c|}
\hline Preoperative pathology & no-NGT $(N=147)$ & NGT $(N=140)$ & total $(\mathrm{N}=287)$ & $P$ value \\
\hline Pathological differentiation & & & & 0.513 \\
\hline Poorly & $64(43.5)$ & $50(35.7)$ & 114(39.7) & \\
\hline Moderately to poorly & 23(15.6) & 25(17.9) & 48(16.7) & \\
\hline Moderately & $37(25.2)$ & 43(30.7) & $80(27.9)$ & \\
\hline Well to moderately and well & $5(3.4)$ & $8(5.7)$ & $13(4.5)$ & \\
\hline Unknown & 18(12.2) & 14(10.0) & $32(11.1)$ & \\
\hline Pathological type & & & & $0.873^{c}$ \\
\hline Adenocarcinoma & $129(87.8)$ & 127(90.7) & 256(89.2) & \\
\hline Signet-ring cell carcinoma & $8(5.4)$ & $6(4.3)$ & $14(4.9)$ & \\
\hline NET & $2(1.4)$ & $1(0.7)$ & $3(1.0)$ & \\
\hline GIST & $8(5.4)$ & $6(4.3)$ & $14(4.9)$ & \\
\hline cTNM $^{a}$ & & & & 0.164 \\
\hline ૧ & $36(24.5)$ & $36(25.7)$ & $72(25.1)$ & \\
\hline ૧ & $32(21.8)$ & $27(19.3)$ & $59(20.6)$ & \\
\hline Q & $62(42.2)$ & $55(39.3)$ & $117(40.8)$ & \\
\hline Q & $7(4.8)$ & $17(12.1)$ & $24(8.4)$ & \\
\hline Others ${ }^{\mathrm{b}}$ & $10(6.8)$ & $5(3.6)$ & $15(5.2)$ & \\
\hline Tumor location & & & & $0.055^{\mathrm{c}}$ \\
\hline Lower & $88(59.9)$ & $67(47.9)$ & $155(54.0)$ & \\
\hline Upper & $30(20.4)$ & 47(33.6) & $77(26.8)$ & \\
\hline Middle & 26(17.7) & $21(15.0)$ & $47(16.4)$ & \\
\hline Total & $3(2.0)$ & $5(3.6)$ & $8(2.8)$ & \\
\hline \multicolumn{5}{|c|}{ NGB nasogastric tube, NET neuroendocrine tumor, GIST gastrointestinal stromal tumor } \\
\hline $\begin{array}{l}\text { a Based on the 8th edition of } \\
\text { Fisher's exact test was applie }\end{array}$ & CC cancer staging s & em. b Non-gastr & adenocarcinom & atients. c \\
\hline
\end{tabular}


Table 1.3

Perioperative information of the patients

\begin{tabular}{|c|c|c|c|c|}
\hline & no-NGT $(N=147)$ & NGT $(N=140)$ & Total $(\mathrm{N}=\mathbf{2 8 7})$ & $P$ value \\
\hline Preoperative treatment & & & & 0.348 \\
\hline No & 78(53.1) & $82(58.6)$ & $160(55.7)$ & \\
\hline Yes & $69(46.9)$ & $58(41.4)$ & $127(44.3)$ & \\
\hline Duration of operation (min) & & & & 0.235 \\
\hline$<240$ & 113(76.9) & $99(70.7)$ & 212(73.9) & \\
\hline$\geq 240$ & $34(23.1)$ & $41(29.3)$ & $75(26.1)$ & \\
\hline Operative approach & & & & $0.531^{c}$ \\
\hline Laparotomy & $66(44.9)$ & $72(51.4)$ & $138(48.1)$ & \\
\hline Laparoscopic-assisted & 15(10.2) & $17(12.1)$ & $32(11.1)$ & \\
\hline Totally Laparoscopic & $63(42.9)$ & $49(35.0)$ & 112(39.0) & \\
\hline Conversion to laparotomy & $3(2.0)$ & $2(1.4)$ & $35(1.7)$ & \\
\hline Resection range & & & & $0.002^{c}$ \\
\hline Distal & $87(59.2)$ & $61(43.6)$ & 148(51.6) & \\
\hline Proximal & $4(2.7)$ & $12(8.6)$ & 16(5.6) & \\
\hline Total & 48(32.7) & $65(46.4)$ & 113(39.4) & \\
\hline Local resection & $8(5.4)$ & $2(1.4)$ & $10(3.5)$ & \\
\hline Multiple organ resection & & & & 0.100 \\
\hline No & 142(96.6) & 129(92.1) & 271(94.4) & \\
\hline Yes & $5(3.4)$ & $11(7.9)$ & 16(5.6) & \\
\hline Lymph node dissection & & & & 0.069 \\
\hline D0 & $9(6.1)$ & $4(2.9)$ & $13(4.5)$ & \\
\hline D1+ & $6(4.1)$ & 14(10.0) & $20(7.0)$ & \\
\hline D2 & 132(89.8) & 122(87.1) & $254(88.5)$ & \\
\hline Surgeon & & & & $0.253^{c}$ \\
\hline \multicolumn{5}{|c|}{ NGB nasogastric tube, ICU intensive care unit } \\
\hline c Fisher's exact test was ap & d. d Continuity Co & ction chi-squar & est was applied. & \\
\hline
\end{tabular}




\begin{tabular}{|c|c|c|c|c|}
\hline & no-NGT $(N=147)$ & NGT $(N=140)$ & Total $(\mathbf{N}=\mathbf{2 8 7})$ & $P$ value \\
\hline A & 122(83.0) & 107(76.4) & 229(79.8) & \\
\hline B & $13(8.8)$ & 13(9.3) & 26(9.1) & \\
\hline C & $10(6.8)$ & 19(13.6) & $29(10.1)$ & \\
\hline Others & $2(1.4)$ & $1(0.7)$ & $3(1.0)$ & \\
\hline The first assistant & & & & 0.438 \\
\hline D & $50(34.0)$ & 44(31.4) & $94(32.8)$ & \\
\hline E & $41(27.9)$ & $37(26.4)$ & 78(27.2) & \\
\hline $\mathrm{F}$ & $40(27.2)$ & $49(35.0)$ & $88(31.0)$ & \\
\hline Others & 16(10.9) & 10(7.1) & 26(9.1) & \\
\hline The degree of adhesion & & & & 0.794 \\
\hline No & 106(72.1) & 102(72.9) & $208(72.5)$ & \\
\hline Mild & 24(16.3) & $25(17.9)$ & $49(17.1)$ & \\
\hline Moderate-severe & 17(11.6) & $13(9.3)$ & $30(10.5)$ & \\
\hline ICU & & & & $0.331^{d}$ \\
\hline No & 143(97.3) & 132(94.3) & $275(95.8)$ & \\
\hline Yes & $4(2.7)$ & $8(5.7)$ & $12(4.2)$ & \\
\hline Blood loss (ml) & & & & 0.713 \\
\hline \multicolumn{5}{|l|}{ Table 1.3(continued) } \\
\hline & no-NGT (N = 147) & NGT $(N=140)$ & Total $(\mathrm{N}=287)$ & $P$ value \\
\hline$<200$ & 115(78.2) & $112(80.0)$ & $227(79.1)$ & \\
\hline$\geq 200$ & $32(21.8)$ & $28(20.0)$ & $60(20.9)$ & \\
\hline \multicolumn{5}{|c|}{ NGB nasogastric tube, ICU intensive care unit } \\
\hline c Fisher's exact test was & ed. d Continuity Cor & ection chi-square & est was applied. & \\
\hline
\end{tabular}

Univariate analysis (Table 2.1$)$ showed that resection range $(p=0.004)$ and lymph node dissection range $(p=0.032)$ were significantly correlated with nasogastric tube placement. These two, together with the preoperative pathological differentiation, clinical TNM stage, operative approach, multiple organ resection, surgeon and nasogastric tube placement required further exploration $(0.05<p<0.2$, 
respectively) were included in the multivariate analysis (Table 2.2), and we found that only resection range $(p=0.004)$ was the independent influence factor of nasogastric tube placement. NGT was omitted in the majority (58.8\%) of distal gastrectomy, but only in $42.5 \%$ and $25 \%$ in total and proximal gastrectomy. 
Table 2.1

Univariate analysis of nasogastric tube placement

\begin{tabular}{|c|c|c|c|c|}
\hline & $\begin{array}{l}\text { no-NGT } \\
(N=147)\end{array}$ & $\begin{array}{l}\text { NGT } \\
(N=140)\end{array}$ & OR $(95 \% \mathrm{Cl})$ & $P$ value \\
\hline Pathological differentiation & & & & 0.517 \\
\hline Poorly & $64(43.5)$ & $50(35.7)$ & Ref & \\
\hline Moderately to poorly & 23(15.6) & 25(17.9) & $1.391(0.707-2.736)$ & 0.339 \\
\hline Moderately & $37(25.2)$ & 43(30.7) & $1.488(0.838-2.642)$ & 0.175 \\
\hline Well to moderately and well & $5(3.4)$ & $8(5.7)$ & $2.048(0.631-6.645)$ & 0.233 \\
\hline Unknown & $18(12.2)$ & $14(10.0)$ & $0.996(0.452-2.194)$ & 0.991 \\
\hline cTNM $^{a}$ & & & & 0.190 \\
\hline$\square$ & $36(24.5)$ & $36(25.7)$ & Ref & \\
\hline ૫ & $32(21.8)$ & 27(19.3) & $0.844(0.423-1.682)$ & 0.629 \\
\hline$\square$ & $62(42.2)$ & $55(39.3)$ & $0.887(0.493-1.596)$ & 0.689 \\
\hline ૫ & $7(4.8)$ & $17(12.1)$ & $2.429(0.899-6.562)$ & 0.080 \\
\hline Others ${ }^{b}$ & $10(6.8)$ & $5(3.6)$ & $0.500(0.155-1.609)$ & 0.245 \\
\hline Operative approach & & & & 0.540 \\
\hline Laparotomy & $66(44.9)$ & $72(51.4)$ & Ref & \\
\hline Laparoscopic-assisted & 15(10.2) & $17(12.1)$ & $1 . .039(0.481-2.245)$ & 0.923 \\
\hline Totally Laparoscopic & $63(42.9)$ & $49(35.0)$ & $0.713(0.432-1.177)$ & 0.186 \\
\hline Conversion to laparotomy & $3(2.0)$ & $2(1.4)$ & $0.611(0.099-3.772)$ & 0.596 \\
\hline Resection range & & & & 0.004 \\
\hline Distal & $87(59.2)$ & $61(43.6)$ & Ref & \\
\hline Proximal & $4(2.7)$ & 12(8.6) & $4.279(1.317-13.896)$ & 0.016 \\
\hline Total & $48(32.7)$ & $65(46.4)$ & $1.931(1.176-3.172)$ & 0.009 \\
\hline Local resection & $8(5.4)$ & $2(1.4)$ & $0.357(0.073-1.737)$ & 0.202 \\
\hline
\end{tabular}

NGB nasogastric tube

a Based on the 8th edition of AJCC cancer staging system. b Non-gastric adenocarcinoma patients. 


\begin{tabular}{|lllll|}
\hline & $\begin{array}{l}\text { no-NGT } \\
(\mathbf{N = 1 4 7 )}\end{array}$ & $\begin{array}{l}\text { NGT } \\
(\mathbf{N}=140)\end{array}$ & OR (95\%Cl) & P value \\
\hline No & $142(96.6)$ & $129(92.1)$ & Ref & \\
\hline Yes & $5(3.4)$ & $11(7.9)$ & $2.422(0.819-7.157)$ & 0.110 \\
\hline Lymph node dissection & & & & 0.081 \\
\hline D2 & $132(89.8)$ & $122(87.1)$ & Ref & \\
\hline D1+ & $6(4.1)$ & $14(10.0)$ & $5.250(1.151-23.937)$ & 0.032 \\
\hline D0 & $9(6.1)$ & $4(2.9)$ & $2.080(0.624-6.927)$ & 0.233 \\
\hline Surgeon & & & & \\
\hline A & $122(83.0)$ & $107(76.4)$ & Ref & 0.751 \\
\hline B & $13(8.8)$ & $13(9.3)$ & $1.140(0.507-2.567)$ & 0.061 \\
\hline C & $10(6.8)$ & $19(13.6)$ & $2.166(0.965-4.863)$ & 0.648 \\
\hline Others & $2(1.4)$ & $1(0.7)$ & $0.570(0.051-6.376)$ & \\
\hline NGB nasogastric tube & & & & \\
\hline a Based on the 8th edition of AJCC cancer staging system. b Non-gastric adenocarcinoma patients. \\
\hline
\end{tabular}

Table 2.2

Multivariate analysis of nasogastric tube placement

\begin{tabular}{|c|c|c|}
\hline & OR $(95 \% \mathrm{Cl})$ & $P$ value \\
\hline Resection range & & 0.004 \\
\hline Distal & Ref & \\
\hline Proximal & $4.555(1.392-14.905)$ & 0.016 \\
\hline Total & $1.990(1.205-3.287)$ & 0.009 \\
\hline Local resection & $0.366(0.074-1.797)$ & 0.202 \\
\hline
\end{tabular}

To further determine the influence of NGT to postoperative recovery, we used PSM to balance the differences between the two groups in resection range. After screened and matched, a total of 230 patients were included, and 115 patients were assigned to the NGT' group and no-NGT' group respectively. The patients characteristics before and after PSM were shown in Table 3. 
Table 3

Clinical characteristics of the patients before and after PSM

\begin{tabular}{|c|c|c|c|c|c|c|}
\hline & \multicolumn{2}{|l|}{ Before } & \multirow[t]{2}{*}{$P$ value } & \multicolumn{2}{|l|}{ After } & \multirow[t]{2}{*}{$P$ value } \\
\hline & $\begin{array}{l}\text { no-NGT } \\
(N=147)\end{array}$ & $\begin{array}{l}\text { NGT } \\
(N=140)\end{array}$ & & $\begin{array}{l}\text { no-NGT' } \\
(\mathrm{N}=115)\end{array}$ & $\begin{array}{l}\text { NGT' }^{\prime} \\
(\mathrm{N}=115)\end{array}$ & \\
\hline Resection range & & & $0.002^{c}$ & & & $1.000^{c}$ \\
\hline Distal & $87(59.2)$ & $61(43.6)$ & & 61(53.0) & $61(53.0)$ & \\
\hline Proximal & $4(2.7)$ & 12(8.6) & & $4(3.5)$ & $4(3.5)$ & \\
\hline Total & $48(32.7)$ & $65(46.4)$ & & $48(41.7)$ & $48(41.7)$ & \\
\hline Local resection & $8(5.4)$ & $2(1.4)$ & & $2(1.7)$ & $2(1.7)$ & \\
\hline \multicolumn{7}{|c|}{ NGB nasogastric tube } \\
\hline c Fisher's exact & t was ap & & & & & \\
\hline
\end{tabular}

In the analysis of the primary endpoint, no statistical differences were found between the two groups (9 days in no-NGT' group versus 10 days in NGT' group, $p=0.344$, Table 4). Non-parametric tests also showed no statistical differences in the other recovery parameters including time to first flatus and defecation, time to fluid and semi-fluid diet, ( $p>0.05$ respectively). The rate of reinsertion and hospitalization expenses between no-NGT' group and NGT' group were not significant either ( $p>0.05$ respectively). 
Table 4

Postoperative recovery and safety parameters

\begin{tabular}{|c|c|c|c|c|}
\hline & $\begin{array}{l}\text { no-NGT' }^{\prime} \\
(\mathrm{N}=115)\end{array}$ & $\begin{array}{l}\text { NGT' }^{\prime} \\
(\mathrm{N}=115)\end{array}$ & $\begin{array}{l}\text { Total } \\
(N=230)\end{array}$ & $P$ value \\
\hline Total hospital stay (d) & $13(5-100)$ & $13(6-75)$ & $13(5-100)$ & 0.407 \\
\hline Postoperative hospital stay (d) & $9(4-98)$ & $10(4-71)$ & $10(4-98)$ & 0.344 \\
\hline First time to ambulation (d) & $1(0-4)$ & $1(0-4)$ & $1(0-4)$ & 0.160 \\
\hline Time to first flatus (d) & $3(1-7)$ & $3(2-32)$ & $3(1-32)$ & 0.903 \\
\hline Time to first defecation (d) & $4(2-10)$ & $4(3-13)$ & $4(2-13)$ & 0.761 \\
\hline Time to fluid (d) & $2(1-6)$ & $2(1-25)$ & $2(1-25)$ & 0.712 \\
\hline Time to semi-fluid (d) & $7(3-96)$ & $8(3-58)$ & $7(3-96)$ & 0.500 \\
\hline Postoperative complications (\%) & & & & 0.753 \\
\hline No & $90(78.3)$ & $88(76.5)$ & 178(77.4) & \\
\hline Yes & $25(21.7)$ & $27(23.5)$ & $52(22.6)$ & \\
\hline Severe complications (\%) & & & & 0.472 \\
\hline No & 107(93.0) & 104(90.4) & $211(91.7)$ & \\
\hline Yes & $8(7.0)$ & $11(9.6)$ & $19(8.3)$ & \\
\hline Infection (\%) & & & & 0.604 \\
\hline No & 108(93.9) & $106(92.2)$ & $214(93.0)$ & \\
\hline Yes & $7(6.1)$ & $9(7.8)$ & $16(7.0)$ & \\
\hline Pulmonary complications (\%) & & & & $0.332^{d}$ \\
\hline No & 108(93.9) & $112(97.4)$ & $220(95.7)$ & \\
\hline Yes & $7(6.1)$ & $3(2.6)$ & $10(4.3)$ & \\
\hline Gastrointestinal obstruction (\%) & & & & $0.622^{c}$ \\
\hline No & 114(99.1) & $112(97.4)$ & $226(98.3)$ & \\
\hline Yes & $1(0.9)$ & $3(2.6)$ & $4(1.7)$ & \\
\hline
\end{tabular}

\footnotetext{
NGB nasogastric tube

c Fisher's exact test was applied. $d$ Continuity Correction chi-square test was applied. Supplementary Data
} 


\begin{tabular}{|c|c|c|c|c|}
\hline & $\begin{array}{l}\text { no-NGT' } \\
(\mathrm{N}=115)\end{array}$ & $\begin{array}{l}\text { NGT' }^{\prime} \\
(\mathrm{N}=115)\end{array}$ & $\begin{array}{l}\text { Total } \\
(N=230)\end{array}$ & $P$ value \\
\hline Delayed gastric emptying (\%) & & & & $0683^{c}$ \\
\hline No & 113(98.3) & $111(96.5)$ & 224(97.4) & \\
\hline Yes & $2(1.7)$ & $4(3.5)$ & $6(2.6)$ & \\
\hline Postoperative hemorrhage (\%) & & & & $0.064^{d}$ \\
\hline No & 106(92.2) & 113(98.3) & 219(95.2) & \\
\hline Yes & $9(7.8)$ & $2(1.7)$ & $11(4.8)$ & \\
\hline Intra-abdominal hemorrhage (\%) & & & & $0.622^{c}$ \\
\hline No & 112(97.4) & 114(99.1) & $226(98.3)$ & \\
\hline Yes & $3(2.6)$ & $1(0.9)$ & $4(1.7)$ & \\
\hline Gastrointestinal hemorrhage (\%) & & & & $0.125^{\mathrm{d}}$ \\
\hline No & $109(94.8)$ & 114(99.1) & 223(97.0) & \\
\hline Yes & $6(5.2)$ & $1(0.9)$ & $7(3.0)$ & \\
\hline Lymphatic leakage (\%) & & & & $0.443^{d}$ \\
\hline No & 113(98.3) & 110(95.7) & 223(97.0) & \\
\hline Yes & $2(1.7)$ & $5(4.3)$ & $7(3.0)$ & \\
\hline Anastomotic leakage (\%) & & & & $1.000^{\mathrm{c}}$ \\
\hline No & $111(96.5)$ & 112(97.4) & $223(97.0)$ & \\
\hline Yes & $4(3.5)$ & $3(2.6)$ & $7(3.0)$ & \\
\hline \multicolumn{5}{|l|}{ Table 4 (continued) } \\
\hline & $\begin{array}{l}\text { no-NGT' } \\
(\mathrm{N}=115)\end{array}$ & $\begin{array}{l}\mathrm{NGT}^{\prime} \\
(\mathrm{N}=115)\end{array}$ & $\begin{array}{l}\text { Total } \\
(\mathrm{N}=230)\end{array}$ & $P$ value \\
\hline Pancreatic leakage (\%) & & & & $0.683^{c}$ \\
\hline No & 113(98.3) & $111(96.5)$ & 224(97.4) & \\
\hline
\end{tabular}

NGB nasogastric tube
c Fisher's exact test was applied. d Continuity Correction chi-square test was applied.
Supplementary Data




\begin{tabular}{|lllll|}
\hline & $\begin{array}{l}\text { no-NGT' } \\
(\mathbf{N}=115)\end{array}$ & $\begin{array}{l}\text { NGT }^{\prime} \\
(\mathbf{N}=115)\end{array}$ & $\begin{array}{l}\text { Total } \\
(\mathbf{N}=230)\end{array}$ & P value \\
\hline Yes & $2(1.7)$ & $4(3.5)$ & $6(2.6)$ & \\
\hline Reinsertion of nasogastric tube (\%) & & & 0.492 \\
\hline No & $103(89.6)$ & $106(92.2)$ & $209(90.9)$ & \\
\hline Yes & $12(10.4)$ & $9(7.8)$ & $21(9.1)$ & \\
\hline Hospitalization expenses(¥) & 90528.00 & 91026.00 & 90919.28 & 0.916 \\
& $(30396.54-$ & $(33583.00-$ & $(30396.54-$ & \\
& $329739.00)$ & $296043.30)$ & $329739.00)$ & \\
\hline NGB nasogastric tube & & & & \\
\hline c Fisher's exact test was applied. d Continuity Correction chi-square test was applied. & & & \\
\hline Supplementary Data & & & \\
\hline
\end{tabular}

The overall complication rate was $24.4 \%$ and the severe complication rate was $8.0 \%$ in the 287 patients before PSM. And those were $22.6 \%$ and $8.3 \%$ respectively after PSM, with no statistical differences between the groups in overall complication rate $(21.7 \%$ versus $23.5 \%, p=0.753)$ or severe complications (7.0\% versus $9.6 \%, p=0.472$, Table 4 ). Comparisons of each respective complication are listed in Table 4 .

\section{Subgroup analysis}

As our multivariate analysis showed that resection range was the only independent factor that influenced the tube placement. We conducted the subgroup analysis in patients with distal gastrectomy and in those with proximal or total gastrectomy, aiming for any factor influencing the tube placement in those two subgroups. In the proximal or total gastrectomy group, we did not find any further independent factor determining the tube placement (See supplementary data, Table S1.1 and S1.2), while in the distal group, the pathological differentiation and operative approach (totally laparoscopic, OR $=0.395,95 \% \mathrm{Cl}=0.187-$ $0.832, p=0.014$ ) were the independent factors that influence the tube placement (See supplementary data, Table S2.1 and S2.2). We also compared the postoperative recovery and safety parameters of the two subgroups, and found no significant differences between patients with or without NGT in neither subgroups (see supplementary data, Table S3, S4.1 and S4.2).

\section{Discussion}

Despite the accumulating data suggesting to omit NGT for gastric cancer surgery, many Chinese centers still routinely apply it when the ERAS guidelines recommend the opposite. Our retrospective analysis of the prospectively maintained database revealed that in our practice, the resection range is the main course of NGT placement. We also found there was not significant difference in postoperative recovery in 
patients with or without NGT in our clinical setting. Our data might help surgeons to have new insights into the practice strategy of ERAS program after gastrectomy.

One major finding of our research is that surgeons' choice of placing NGT is mainly influenced by the resection range, i.e. proximal and total gastrectomy. NGT is more often placed when the surgeon estimates a high risk of the surgical approach which might eventually result in anastomotic or other complications after surgery [16]. Importantly, it turned out that those two resection ranges were indeed had higher complication rates when compared to distal gastrectomy (data not shown). In the latter group, NGT was omitted in the majority (58.8\%), with satisfactory recovery and safety outcomes. From this point of view, our data demonstrate that the surgeons' decision-making of NGT placement is, at least, reasonable.

However, our data also suggest such effort of NGT placement in high-risk patients did not result in better safety outcomes. In this prospective registry, the complication rates (overall $28 \%$ ) were comparable to the other studies [17]. For proximal or total gastrectomy, doctors choose to insert the nasogastric tube partly because the decompression effect may prevent leakage into the thoracic cavity, but our results do not indicate additional benefits (i.e. lower complication rate) of such attempt. Similarly, omitting NGT in the distal ones did not increase the complication rate either based on our subgroup analysis. Our data therefore confirmed the safety of omitting NGT in all types of gastrectomy.

As one critical element in the ERAS programs, many studies report the bowel function recovery as main outcomes to investigate whether NGT placement delays its recovery. One commonly used parameter is the time to first flatus. Varied results were reported regarding whether NGT shortens the time to first flatus [18]. Our data, in accordance with the meta-analyses $[3,19]$ and the prospective randomized controlled trial [10], showed no effect in this regard. However, it is important to notice that actually the time to flatus is not a good indicator of the bowel function recovery [20], let alone its occurrence is quite subjective in clinical practice. In contrast, the study by van Bree [21] indicates that tolerance of solid food and first defecation are better indicator of bowel function recovery instead. Due to a difference in postoperative diet between Chinese and western culture, time to semi-liquid instead of solid food is used to indicate the bowel function in our study. But no difference was found in either time to semi-liquid or time to first defecation in our study. Similar results were also reported by Hu et. al. [22], although many other studies reported earlier defecation in the no-NGT group $[23,24]$.

On top of bowel function recovery, the ultimate purpose of the ERAS program is to yield a safe and faster recovery after surgery, hence we chose the postoperative stay, instead of the bowel recovery outcomes, as our primary endpoint in this study. Unfortunately, the good safety outcomes in the no-NGT didn't result in shorter hospital stay (e.g. postoperative stay, prolonged hospitalization) in our analysis. This is probably because of the fact that omitting NGT is only one small procedure in the concept of ERAS and itself may not have enough influence to the whole picture, especially when an ERAS protocol had been running routinely in our practice. Of course, our study is also subject to its retrospective nature and limited to a relatively small group size. These limitations may also influence the statistical power of our results. 


\section{Conclusions}

In this retrospective analysis of our prospectively maintained database, we found that the decisionmaking of nasogastric tube placement was mainly influenced by the resection range in gastric cancer surgery. Omitting NGT is a safe approach in all types of gastrectomy but was not able to enhance the recovery in our practice.

\section{Abbreviations}

\section{ERAS}

Enhanced Recovery after Surgery

NGB

Nasogastric Tube

RCT

Randomized Controlled Trial

PCA

Patient-controlled Analgesia

PSM

Propensity Score Matching

BMI

Body Mass Index

NRS

Nutritional Risk Screening

NET

Neuroendocrine Tumor

GIST

Gastrointestinal Stromal Tumor

ICU

Intensive Care Unit

pPLOS

prolonged Postoperative Length of Stay

\section{Declarations}

Ethics approval and consent to participate

The current study is approved by Peking University Cancer Hospital Ethics Committee (2016YJZ32). This study is a retrospective analysis for our prospectively maintained database. It does not require permission/consent of the participants.

Consent for publication 
Not applicable.

\section{Availability of data and materials}

The datasets generated and/or analysed during the current study are not publicly available because they are derived from the patient database of the center and hence subject to confidentiality but are available from the corresponding author on reasonable request.

\section{Competing interests}

The authors declare that they have no competing interests.

\section{Funding}

This study is funded by the Beijing Municipal Science \& Technology project, Beijing Municipal science \& technology commission (D131100005313010), and National Key Technology Research and Development Program of the Ministry of Science and Technology of China (2014BAI09B02). Neither of them was involved in the study design, data collection, analysis and interpretation of the data, or manuscript writing.

\section{Authors' contributions}

WZQ and LZY conceived of the study. WZQ and WQ were the major contributors in drafting and revising the manuscript. WQ, SJY and HSY collected patients' data. WQ and ZY provided statistical analysis and interpretation. JJF, LZY, SF and LSX provided necessary administrative support for the study. All authors contributed to refinement of the study protocol and approved the final manuscript.

\section{Acknowledgements}

Not applicable.

\section{References}

[1] Yang Z, Zheng Q, Wang Z. Meta-analysis of the need for nasogastric or nasojejunal decompression after gastrectomy for gastric cancer. Br J Surg. 2008;95:809-16.

[2] Verma R, Nelson RL. Prophylactic nasogastric decompression after abdominal surgery. Cochrane Database Syst Rev. 2007;5:CD004929.

[3] Ding J, Liao G, Xia Y, Zhang ZM, Pan Y, Liu S, et al. The necessity of indwelling gastrointestinal decompression after gastrectomy: a meta-analysis. J Surg Res. 2013;179:e71-e81.

[4] Mortensen K, Nilsson M, Slim K, Schäfer M, Mariette C, Braga M, et al. Consensus guidelines for enhanced recovery after gastrectomy: Enhanced Recovery After Surgery (ERAS $®$ ) Society recommendations. Br J Surg. 2014;101:1209-29. 
[5] Robotic and Laparoscopic Surgery Committee of Chinese Research Hospital Association. Expert consensus on enhanced recovery after gastrectomy for gastric cancer (2016 edition). Chin J Dig Surg. 2017;16:14-7.

[6] Carrère N, Seulin P, Julio CH, Bloom E, Gouzi JL, Pradère B. Is nasogastric or nasojejunal decompression necessary after gastrectomy? A prospective randomized trial. World J Surg. 2007;31:1227.

[7] Doglietto GB, Papa V, Tortorelli AP, Bossola M, Covino M, Pacelli F. Nasojejunal tube placement after total gastrectomy: a multicenter prospective randomized trial. Arch Surg. 2004;139:1309-13; discussion 1313.

[8] Hsu SD, Yu JC, Chen TW, Chou SJ, Hsieh HF, Chan DC. Role of Nasogastric Tube Insertion after Gastrectomy. Chir Gastroenterol. 2007;23:303-6.

[9] Li C, Mei JW, Yan M, Chen MM, Yao XX, Yang QM, et al. Nasogastric decompression for radical gastrectomy for gastric cancer: a prospective randomized controlled study. Dig Surg. 2011;28:167-72.

[10] Yoo CH, Son BH, Han WK, Pae WK. Nasogastric decompression is not necessary in operations for gastric cancer: prospective randomised trial. Eur J Surg. 2002;168:379-83.

[11] Chen K, Mou YP, Xu XW, Xie K, Zhou W. Necessity of routine nasogastric decompression after gastrectomy for gastric cancer: a meta-analysis. Zhonghua Yi Xue Za Zhi. 2012;92:1841-4.

[12] Shi J, Wu Z, Li Z, Ji J. Drainage in the first postoperative days predicts intra-abdominopelvic complications, International Journal of Surgery (Chinese), 2018;45:233-7

[13] Komen N, Slieker J, Willemsen P, Mannaerts G, Pattyn P, Karsten T, et al. Acute phase proteins in drain fluid: a new screening tool for colorectal anastomotic leakage? The APPEAL study: analysis of parameters predictive for evident anastomotic leakage. Am J Surg. 2014;208:317-23.

[14] Komen N, Slieker J, Willemsen P, Mannaerts G, Pattyn P, Karsten T, et al. Polymerase chain reaction for Enterococcus faecalis in drain fluid: the first screening test for symptomatic colorectal anastomotic leakage. The Appeal-study: analysis of parameters predictive for evident anastomotic leakage. Int J Colorectal Dis. 2014;29:15-21.

[15] Dindo D, Demartines N, Clavien PA. Classification of surgical complications: a new proposal with evaluation in a cohort of 6336 patients and results of a survey. Ann Surg. 2004;240:205-13.

[16] Wang D, Li T, Yu J, Hu Y, Liu H, Li G. Is nasogastric or nasojejunal decompression necessary following gastrectomy for gastric cancer? A systematic review and meta-analysis of randomised controlled trials. J Gastrointest Surg. 2015;19:195-204. 
[17] Lee KG, Lee HJ, Yang JY, Oh SY, Bard S, Suh YS, et al. Risk factors associated with complication following gastrectomy for gastric cancer: retrospective analysis of prospectively collected data based on the Clavien-Dindo system. J Gastrointest Surg. 2014;18:1269-77.

[18] Pacelli F, Rosa F, Marrelli D, Morgagni P, Framarini M, Cristadoro L, et al. Naso-gastric or naso-jejunal decompression after partial distal gastrectomy for gastric cancer. Final results of a multicenter prospective randomized trial. Gastric Cancer. 2014;17:725-32.

[19] Wei ZW, Li JL, Li ZS, Hao YT, He YL, Chen W, et al. Systematic review of nasogastric or nasojejunal decompression after gastrectomy for gastric cancer. Eur J Surg Oncol. 2014;40:1763-70.

[20] Wu Z, Boersema GS, Dereci A, Menon AG, Jeekel J, Lange JF. Clinical endpoint, early detection, and differential diagnosis of postoperative ileus: a systematic review of the literature. Eur Surg Res. 2015;54:127-38.

[21] van Bree SH, Bemelman WA, Hollmann MW, Zwinderman AH, Matteoli G, El Temna S, et al. Identification of clinical outcome measures for recovery of gastrointestinal motility in postoperative ileus. Ann Surg. 2014;259:708-14.

[22] Hu JX, Li LL, Dai F, Wu HY. Clinical observation of radical total gastrectomy without postoperative gastrointestinal decompression in elderly patients with gastric cancer. Int J Nurs Sci. 2015;2:47-51.

[23] Feng K. Evaluation of nasogastric decompresion in postoperative gastric cancer patients:a prospective randomized trial. J Dig Oncol (Electronic Version). 2009;1:105-8.

[24] Wang LH, Zhu RF, Gao C, Wang SL, Shen LZ. Application of enhanced recovery after gastric cancer surgery: An updated meta-analysis. World J Gastroenterol. 2018;24:1562-78.

\section{Figures}




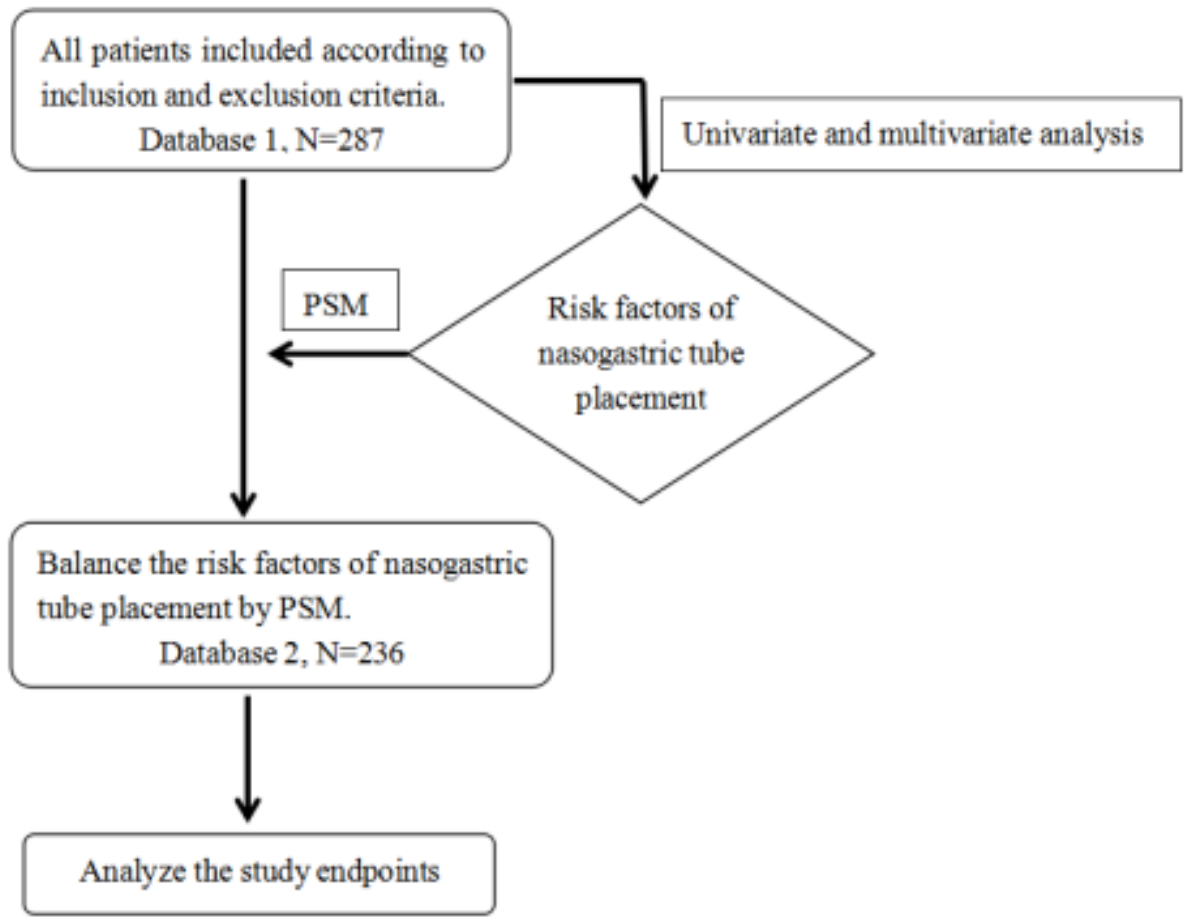

Fig.1 Flow chart of the study

\section{Figure 1}

Flow chart of the study

\section{Supplementary Files}

This is a list of supplementary files associated with this preprint. Click to download.

- SupplementaryData.docx 